\title{
Viroimmunotherapy for breast cancer: promises, problems and future directions
}

\author{
Shyambabu Chaurasiya $\mathbb{( i b}^{1} \cdot$ Yuman Fong ${ }^{1}$
}

Received: 18 May 2020 / Revised: 26 August 2020 / Accepted: 12 November 2020 / Published online: 2 December 2020

(c) The Author(s), under exclusive licence to Springer Nature America, Inc. 2020

\begin{abstract}
Virotherapy, a strategy to use live viruses as therapeutics, is a relatively novel field in the treatment of cancer. With the advancements in molecular biology and virology, there has been a huge increase in research on cancer virotherapy. For the treatment of cancer, viruses could be used either as vectors in gene therapy or as oncolytic agents. A variety of viruses have been studied for their potential usage in gene therapy or oncolytic therapy. In this review, we discuss virotherapy with a special focus on breast cancer. Breast cancer is the most common cancer and the leading cause of cancer-related deaths in women worldwide. Current treatments are insufficient to cure metastatic breast cancer and are often associated with severe side effects that further deteriorates patients' quality of life. Therefore, novel therapeutic approaches such as virotherapy need to be developed for the treatment of breast cancer. Here we summarize the current treatments for breast cancer and the potential use of virotherapy in the treatment of the disease. Furthermore, we discuss the use of oncolytic viruses as immunotherapeutics and the rational combination of oncolytic viruses with other therapeutics for optimal treatment of breast cancer. Finally, we outline the progress made in virotherapy for breast cancer and the shortcomings that need to be addressed for this novel therapy to move to the clinic for better treatment of breast cancer.
\end{abstract}

\section{Breast cancer}

Breast cancer $(\mathrm{BrCa})$ is the second most commonly diagnosed cancer in the world and according to the last Global Cancer Statistics (GLOBOCON 2018), BrCa represented $11.6 \%$ of all cancers and caused $6.6 \%$ of all cancer-related deaths [1]. Major advancements in the diagnosis and treatment of $\mathrm{BrCa}$ made over the last three decades have improved the quality of life and overall survival of patients. However, despite improvements in diagnostic methods, $20-50 \%$ of patients develop metastatic disease which remains difficult to treat and accounts for the majority of deaths $[2,3]$. $\mathrm{BrCa}$ is a highly heterogeneous disease, and the extensive intra- and inter-tumoral heterogeneity are thought to be the result of the distinct cells of origin and specific alterations at genetic and epigenetic levels [4]. $\mathrm{BrCa}$ has been classified in many ways and the classification is

Shyambabu Chaurasiya

schaurasiya@coh.org

1 Department of Surgery, City of Hope National Medical Center, Duarte, CA, USA ever-evolving. Traditional classifications of $\mathrm{BrCa}$ were based on the histology and biology of the tumors, whereas recent classification schemes are mainly based on molecular differences [5, 6]. Comprehensive gene expression profiling by different independent groups using a large set of breast tumors has led researchers to categorize $\mathrm{BrCa}$ into five major molecular sub-types, which vary in the expression levels of the estrogen receptor (ER) and human epidermal growth factor receptor 2 (HER2). The five molecular subtypes are normal breast-like, luminal A, luminal B, HER2 ${ }^{+} / \mathrm{ER}^{-}$, and basal-like or triple-negative [7, 8]. Treatments for $\mathrm{BrCa}$ are dictated to some extent by the molecular subtypes and prognosis varies among the sub-types, but in general, patients with basal-like (or triple-negative) tumors have the worst prognosis whereas patients with luminal A tumors have the best prognosis $[4,9]$.

\section{Current therapies}

Surgery is commonly used to remove primary breast tumors while radiation and chemotherapies are used as adjuvants in the treatment of $\mathrm{BrCa}[2,10]$. For most of the twentieth century, the Halsted radical mastectomy, an aggressive form 
of surgery that includes removal of the breast together with muscles of the chest wall, was the standard form of surgery for the treatment of $\mathrm{BrCa}[11,12]$. This approach was based on the premise that metastasis in $\mathrm{BrCa}$ occurs through the locoregional spread and thus could be cured by aggressive surgery. However, studies in the 1970s showed that radical mastectomy has no benefit over less aggressive surgeries in terms of either disease recurrence or overall survival $[11,13]$. Later, less aggressive breast conservation surgery became more common for local therapy combined with a wide variety of systemic adjuvant therapies [14]. Adjuvant therapies given systemically are aimed at eradicating disseminated cancer cells in order to minimize the probability of metastatic growth [15]. Like many other types of cancer, metastases are the main cause of death in $\mathrm{BrCa}$ patients, accounting for more than $90 \%$ of total mortality [16]. Studies have shown that the use of adjuvant radiation-, chemo-, endocrine and HER2-directed therapies significantly reduces the risk of disease recurrence and improves the overall survival of patients [17-19]. Radiation is usually applied to the tumor bed and regional lymph nodes after the resection of the primary tumor [20]. The most common chemotherapy adjuvant regimens include the combination of cyclophosphamide with doxorubicin and/or docetaxel or methotrexate with 5-fluorouracil (an excellent review on this topic is provided by Anampa et al. [13]). However, the use of radiation- and chemotherapies is limited by off-target toxicities and development of resistance to these therapies $[21,22]$. Off-target toxicities resulting from chemotherapies substantially affect the quality of patients' lives and $\sim 40 \%$ of patients suffer from disease recurrence and die of metastases [23]. More recent therapies for $\mathrm{BrCa}$ have been developed to target the expression of estrogen receptor (ER), progesterone receptor (PR) and human epidermal growth factor receptor 2 (HER2) expression. About $75 \%$ of human BrCa express ER, and endocrine therapy is used for the treatment of such subtype [24]. Tamoxifen, an ER modulator, is the most commonly used endocrine therapy for $\mathrm{ER} / \mathrm{PR}^{+} \mathrm{BrCa}$. Patients with $\mathrm{HER} 2^{+} \mathrm{BrCa}$ are treated with trastuzumab, a monoclonal antibody against HER2. Trastuzumab is usually given in combination with adjuvant chemotherapy either concurrently or sequentially [25]. However, trastuzumab has been shown to induce some degree of cardiac toxicity, and approximately half of the treated patients experience disease relapse within 3 years [26, 27].

Basal-like or triple-negative breast cancer (TNBC) is the most aggressive subtype with the highest relapse rate and high incidence of brain metastasis [28]. TNBCs are not amenable to currently available targeted therapies as they lack these molecular targets. Anthracyclines and taxanes are usually used in the treatment of TNBC, however with very limited success [28]. Although favorable responses to chemotherapy are initially observed in TNBC patients, they quickly develop resistance to the drugs, and disease relapse is very common with the median survival of only 13 months [29]. Taken together, with the advancements in knowledge about BrCa many therapeutic options have been developed, which have substantially increased the overall survival of patients, however, there is still a need for better treatment options to further improve patients' quality of life as well as overall survival.

\section{Virotherapy for breast cancer}

During the course of evolution, cancer cells accumulate many mutations that confer the cells with the ability to resist cell death, acquire replicative immortality, and render them insensitive to immune surveillance [30]. Interestingly, many of the same mutations that allow cancer cells to thrive, also make them better hosts for viruses. Virotherapy, a strategy to use live viruses as therapeutics, exploits these mutated loopholes to target cancer cells. Cancer virotherapy could be broadly categorized into two sub-classes: (i) use of nonreplicating viruses as vectors in cancer gene therapy and (ii) use of replicating viruses as oncolytic agents. In this review article, we will discuss both aspects of virotherapy with respect to $\mathrm{BrCa}$.

\section{Non-replicating viruses for breast cancer therapy}

Many preclinical studies as well as clinical trials have examined the feasibility of gene therapy for the treatment of $\mathrm{BrCa}$. Unlike gene therapy for monogenic disorders where the goal is to restore the function of the defective gene, the goal of cancer gene therapy is to directly or indirectly kill cancer cells [31]. For direct killing of cancer cells, the strategy of suicide gene therapy is employed, which involves the delivery of a toxic gene or a gene that could convert an inactive prodrug into a cytotoxic drug specifically in cancer cells causing cell death [32]. Two most studied strategies for suicide gene therapy are (i) herpes simplex virus (HSV)-thymidine kinase (TK) gene in combination with prodrug ganciclovir and (ii) cytosine deaminase gene in combination with the prodrug 5-fluorocytosine [32].

Majumdar et al. [33] studied adenovirus-mediated HSVTK (Ad-HSV-TK) expression plus ganciclovir as suicide gene therapy in BrCa models. The authors found that HSVTK-ganciclovir therapy had a minimal effect against the murine 4T1 tumors, however, a combination of HSV-TKganciclovir therapy with adenoviruses expressing interleukin(IL)-2 and granulocyte-macrophage colony-stimulating factor (GM-CSF) resulted in substantial anti-tumor 
effect [33]. Similar findings were published by another group using the same vectors and prodrug in a BrCa model [34]. The first clinical trial of a suicide gene therapy for BrCa was reported in 1999 by Pandha and colleagues [35]. In this trial, a plasmid encoding cytosine deaminase under the transcriptional control of an erbB-2 promoter sequence was injected intra-tumorally in patients with erbB-2overexpressing tumors and the prodrug 5-fluorocytosine was administered systemically. This phase I trial showed that the treatment was safe and resulted in targeted expression of the gene in the majority of subjects. However, the study failed to achieve an appreciable anti-tumor effect [35]. Another Phase I trial involving prodrug and gene therapy for BrCa was reported by Braybrooke et al. in 2005 [36]. This study used a retrovirus encoding human cytochrome P450 gene (MetXia-P450) for direct injection into metastatic cutaneous tumor nodules followed by oral administration of the prodrug cyclophosphamide. Treatment was found to be safe and viral gene expression was detected in 10 out of 12 patients. Some levels of anti-tumor efficacy were observed in a fraction of patients and the authors suggested that MetXia-P450 should undergo further clinical assessment. However, no further trial has been reported after the first study on MetXia-P450. According to clinicaltrials.gov (accessed: July 2020) a phase II trial aimed at evaluating the combination of radiation therapy with suicidal gene therapy followed by immunotherapy (checkpoint inhibitor, pembrolizumab), is currently recruiting patients with TNBC or non-small cell lung cancer (NCT03004183). This multi-pronged therapeutic strategy will use Ad-HSVTK virus and the prodrug valacyclovir. It remains to be seen how gene therapy in combination with radiation and immunotherapy works for TNBC patients.

Another common strategy of cancer gene therapy is to use viral vectors armed with toxic genes or other genes such as cytokines, whose products directly or indirectly kill tumor cells. A plethora of preclinical studies on this approach have been reported in the context of $\mathrm{BrCa}$, and some of them have also been evaluated in clinical trials. One such cytotoxic gene is TNF-related apoptosis-inducing ligand (TRAIL) that has been intensively studied for its potential use in gene therapy. A study by Zheng et al. [37] found that an adeno-associated virus encoding soluble TRAIL could efficiently suppress the growth of breast tumors of human origin in nude mice. Likewise, a study by Lin et al. showed that adenovirus-mediated TRAIL gene therapy was effective in killing different types of BrCa cells both in vitro and in vivo and that the combination of TRAIL gene therapy with chemotherapies resulted in additive/ synergistic anti-tumor effects [37]. Additionally, many other proapoptotic genes have been studied for $\mathrm{BrCa}$ gene therapy including Bik [38], BAX [39] and Fas ligands [40]. Likewise, a variety of immune-stimulatory cytokines including GM-CSF [41], IL-2 [42], IL-12 [43], and RANTES [44] have been used as transgenes in $\mathrm{BrCa}$ gene therapy. While most of the preclinical studies found gene therapy to be effective against $\mathrm{BrCa}$, only a handful of them have been tested in clinical trials and the results are not overly convincing.

\section{Replicating viruses for breast cancer therapy}

Oncolytic viruses (OV) represent a class of novel biotherapeutics which can specifically replicate in and kill cancer cells while leaving normal cells unharmed. Although the concept of the oncolytic virus is not new, the field is gaining momentum only recently as advancements in technology have made it feasible to thoroughly study viruses and analyze the safety as well as their anti-tumor efficacy [45]. In the last two decades, many preclinical studies have reported promising anti-tumor effects of a wide range of OVs, many of which have entered clinical trials. Recently the first OV (outside China) Talimogene Laherprepvac ( $\mathrm{T}-\mathrm{Vec}$ ), a herpes simplex virus encoding GMCSF, was approved by the FDA for the treatment of metastatic melanoma [46].

$\mathrm{BrCa}$ is one of the commonly studied cancers for investigating oncolytic viruses in preclinical studies. Many types of viruses have been studied for their oncolytic properties in $\mathrm{BrCa}$ in preclinical (Table 1) and clinical settings (Table 2), and adenovirus is one of the most commonly studied viruses as an oncolytic agent. Oncolytic adenoviruses are targeted to cancer using two main strategies: transductional targeting that allows the virus to specifically infect cancer cells, and transcriptional targeting that allows the virus to replicate specifically in cancer cells [47].

A study published by Bauerschmitz et al. [48], utilized both transductional and transcriptional targeting to restrict the oncolytic activity of an adenovirus to cancer stem-like cells $\left(\mathrm{CD} 44^{+} \mathrm{CD} 24^{-}\right)$isolated from the pleural effusion of BrCa patients. For transductional targeting, the authors used chimeric fiber protein from serotype 3 and 5 of human adenovirus and compared several different promoters for their ability to restrict the expression of E1a, a crucial gene for virus replication, to $\mathrm{BrCa}$ cells. Among different promoters studied for transcriptional targeting, promoters from cyclo-oxygenase- 2 and multi-drug resistance genes were found to be the most active in $\mathrm{CD} 44^{+} \mathrm{CD} 24^{-}$population of $\mathrm{BrCa}$ and the adenovirus constructs with E1a gene controlled by these promoters were able to eradicate the cells in vitro and showed anti-tumor efficacy in vivo in mice. Likewise, Liikanen et al. [49] reported another oncolytic adenovirus transductionally targeted through chimeric fiber and engineered to encode a full-length antibody against HER2. The virus was found to effectively kill $\mathrm{HER} 2^{+} \mathrm{BrCa}$ 
Table 1 Oncolytic viruses tested against breast cancer in preclinical studies.

\begin{tabular}{|c|c|c|c|}
\hline Virus & Modification & Combination & References \\
\hline Adenovirus & Fiber modified; tissue-specific promoter-driven E1A & None & Bauerschmitz et al. [48] \\
\hline Adenovirus & $\begin{array}{l}\text { Chimeric fiber protein; deletion in E1A gene and } \\
\text { encodes trastuzumab }\end{array}$ & None & Liikanen et al. [49] \\
\hline Adenovirus & $\begin{array}{l}\text { E1A under control of hTERT and E1B under control of } \\
\text { HRE promoter; encodes IL- } 24\end{array}$ & None & Zhu et al. [92] \\
\hline Adenovirus & Modified fiber protein; encodes TGF- $\beta$ decoy protein & Anti-PD-L1, anti-CTLA-4 & $\mathrm{Xu}$ et al. [50] \\
\hline HSV-1 & $\begin{array}{l}\text { Re-targeted to HER } 2 \text { by trastuzumab in } \mathrm{gD} \text { domain; } \\
\text { deletion of } \gamma 34.5\end{array}$ & None & Menotti et al. [52] \\
\hline HSV-1 & Deletions in $\alpha 47, \gamma 34.5$, and ICP6 genes; encodes IL-12 & None & Ghouse et al. [54] \\
\hline VACV & $\begin{array}{l}\text { Deleted of J2R, F14.5L, and A56R, and armed with } \\
\text { scAb against VEGF }\end{array}$ & None & Gholami et al. [55] \\
\hline VACV & J2R gene deleted; encodes IL-15 & PI3Kס inhibitor & Ferguson et al. [56] \\
\hline VACV & $\mathrm{J} 2 \mathrm{R}$ replaced with an expression cassette for GM-CSF & Anti-PD-L1, anti-CTLA-4 & Chon et al. [57] \\
\hline VACV & Targeted deletion of immune-modulatory genes & None & Umer et al. [58] \\
\hline Chimeric poxvirus & Deletions in J2R and F14.5L genes; encodes hNIS gene & Anti-PD-L1 & Chaurasiya et al. [87] \\
\hline VSV & Point mutation in matrix protein & Inactivated cancer cells & Niavarani et al. [59] \\
\hline VSV & Mutation in matrix protein & Trastuzumab emtansine & Arulanandam et al. [60] \\
\hline Maraba virus & Mutations in $\mathrm{G}$ and $\mathrm{M}$ protein & Anti-PD-L1, anti-CTLA-4 & $\begin{array}{l}\text { Bourgeois-Daigneault } \\
\text { et al. [61] }\end{array}$ \\
\hline Maraba virus & Mutations in $\mathrm{G}$ and $\mathrm{M}$ protein & Paclitaxel & $\begin{array}{l}\text { Bourgeois-Daigneault } \\
\text { et al. [62] }\end{array}$ \\
\hline NDV & $\begin{array}{l}\text { IL-12 expression cassette inserted between } M \text { and } \\
\text { F genes }\end{array}$ & None & Amin et al. [63] \\
\hline Reovirus & $\begin{array}{l}\text { Reassorted reovirus by forward genetics for enhanced } \\
\text { infectivity }\end{array}$ & Topoisomerase inhibitors & Stewart et al. [64] \\
\hline $\begin{array}{l}\text { VSV, Reovirus, } \\
\text { HSV, and Ad }\end{array}$ & Different modifications in different viruses & Surgery & Martin et al. [91] \\
\hline
\end{tabular}

$V S V$ vesicular stomatitis virus, $V A C V$ vaccinia virus, $A d$ adenovirus, $H R E$ hypoxia response element, $P D-L 1$ programmed death-ligand-1, $C T L A-4$ cytotoxic T lymphocyte antigen $4, H S V$ herpes simplex virus, $h N I S$ human sodium iodide symporter, $P I 3 K \delta$ phosphatidylinositol-3-kinase subunit $\delta, T N B C$ triple-negative breast cancer, $N D V$ Newcastle disease virus, GM-CSF granulocyte-macrophage colony-stimulating factor, $I L-12$ interleukin-12.

cells in vitro and resulted in a significant anti-tumor effect against a HER2 ${ }^{+}$tumor in mice. Another oncolytic adenovirus for $\mathrm{BrCa}$ was studied by $\mathrm{Xu}$ et al. [50], in which the fiber protein was altered to include Lyp-1 peptide to reduce hepatic toxicity and increase infection of $\mathrm{BrCa}$ cells, which usually express high levels of Lyp-1 receptor. Furthermore, the virus was engineered to encode a decoy of transforming growth factor-beta (TGF- $\beta$ ). The virus was found to induce a potent anti-tumor response in murine TNBC models, and it augmented anti-PD-L1 and anti-CTLA-4 therapy.

Other DNA viruses commonly studied for their oncolytic usage are HSV and Vaccinia virus (VACV). Unlike adenovirus that has a specific receptor through which it enters the cell, entry of HSV is complex and cellular receptors for HSVs are not well defined [51]. Because of the lack of specific receptor, HSVs are often targeted at transcriptional levels and not at transductional levels. However, there are some reports suggesting that it is possible to de-target HSV from their natural receptor and retarget them to cancerspecific receptors. In 2008, Menotti et al. [52] constructed an HSV-1 that was fully re-targeted to HER2 protein. This virus was found to enter cells solely via the HER2 receptor. Later, the same group reported the oncolytic potential of this virus in ovarian and $\mathrm{BrCa}$ models. The virus was delivered systemically in mice, using mesenchymal stromal cells as a carrier, bearing HER $2^{+}$tumor xenografts. The authors showed that the virus inhibited growth of primary tumors and reduced $\mathrm{BrCa}$ metastases to the brain [53]. Another oncolytic HSV was tested against a preclinical TNBC model by Ghouse et al. [54]. This virus was modified to encode the cytokine IL-12 and was deleted in three genes $(\alpha 47, \gamma 34.5$, and ICP6) to increase cancer selectivity. In a murine model of TNBC, the virus was found to reduce primary tumor burden and metastasis, and the virus-encoded IL-12 resulted in inhibition of tumor angiogenesis. 


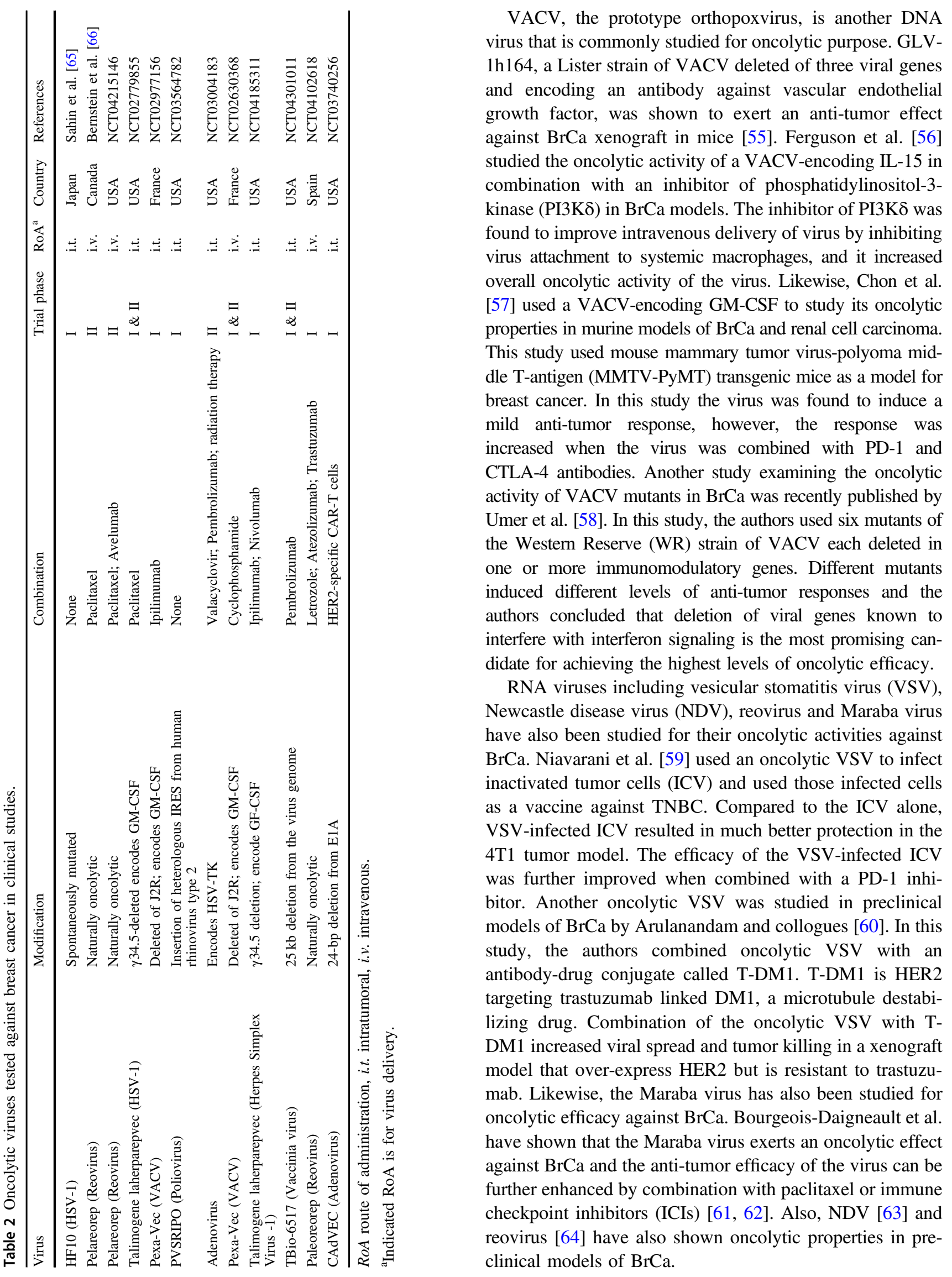

VACV, the prototype orthopoxvirus, is another DNA virus that is commonly studied for oncolytic purpose. GLV1h164, a Lister strain of VACV deleted of three viral genes and encoding an antibody against vascular endothelial growth factor, was shown to exert an anti-tumor effect against $\mathrm{BrCa}$ xenograft in mice [55]. Ferguson et al. [56] studied the oncolytic activity of a VACV-encoding IL-15 in combination with an inhibitor of phosphatidylinositol-3kinase (PI3K $\delta$ ) in $\mathrm{BrCa}$ models. The inhibitor of PI3K $\delta$ was found to improve intravenous delivery of virus by inhibiting virus attachment to systemic macrophages, and it increased overall oncolytic activity of the virus. Likewise, Chon et al. [57] used a VACV-encoding GM-CSF to study its oncolytic properties in murine models of $\mathrm{BrCa}$ and renal cell carcinoma. This study used mouse mammary tumor virus-polyoma middle T-antigen (MMTV-PyMT) transgenic mice as a model for breast cancer. In this study the virus was found to induce a mild anti-tumor response, however, the response was increased when the virus was combined with PD-1 and CTLA-4 antibodies. Another study examining the oncolytic activity of VACV mutants in $\mathrm{BrCa}$ was recently published by the Western Reserve (WR) Stain of VACV each deleted in one or more immunomodulatory genes. Different mutants induced different levels of anti-tumor responses and the authors concluded that deletion of viral genes known to interfere with interferon signaling is the most promising candate for achieving the highest levels of oncolytic efficacy.

vesicular stomatitis virus (VSV), have also been studied for their oncolytic activities against BrCa. Niavarani et al. [59] used an oncolytic VSV to infect inted cells VSV-infected ICV resulted in much better protection in the 4T1 tumor model. The efficacy of the VSV-infected ICV was further improved when combined with a PD-1 inhibitor. Another oncolytic VSV was studied in preclinical models of $\mathrm{BrCa}$ by Arulanandam and collogues [60]. In this study, the authors combined oncolytic VSV with an ntibody-drug conjugate called T-DM1. T-DM1 is HER2 argeting trastuzumab linked DM1, a microtubule destabiizing drug. Combination of the oncolytic VSV with TDM1 increased viral spread and tumor killing in a xenograft model that over-express HER2 but is resistant to trastuzumab. Likewise, the Maraba virus has also been studied for oncolytic efficacy against $\mathrm{BrCa}$. Bourgeois-Daigneault et al. have shown that the Maraba virus exerts an oncolytic effect against $\mathrm{BrCa}$ and the anti-tumor efficacy of the virus can be further enhanced by combination with paclitaxel or immune checkpoint inhibitors (ICIs) [61, 62]. Also, NDV [63] and clinical models of $\mathrm{BrCa}$. 


\section{Clinical studies of virotherapy for breast cancer}

Many types of virotherapy have been tested against $\mathrm{BrCa}$ in different phases of clinical trials either as monotherapy or in combination with other drugs. In 2012, Shahin et al. [65] reported findings from a phase I trial of an oncolytic HSV-1 called HF10. Only six patients with recurrent $\mathrm{BrCa}$ were enrolled in this study. In this study, within the same patient, one tumor nodule was injected with the virus and another tumor nodule was injected with mock control. The virus was found to be well tolerated (maximum dose used was three injections of $5 \mathrm{e}^{05}$ plaque-forming units (PFU)), virus replication was observed in the tumor and virus-treated tumors had significantly higher numbers of $\mathrm{CD}^{+} \mathrm{T}$ cells compared to mock-treated tumors. From this study, the authors concluded that HF10 was a promising candidate for further clinical evaluations, however, no clinical trials have been announced yet for this virus for the treatment of $\mathrm{BrCa}$. In 2017, Bernstein et al. [66] reported the results of a phase II trial of oncolytic reovirus (Pelareorep) in combination with paclitaxel in patients with metastatic BrCa. A total of 74 patients were enrolled in this Canadian study. Both the virus and Paclitaxel were injected intravenously. While the combination treatment resulted in a significant increase in the overall survival compared to Paclitaxel alone treatment, there was no significant difference in progression-free survival of patients treated with Paclitaxel alone or in combination with Pelareorep. There is an active Phase II trial currently recruiting $\mathrm{BrCa}$ patient to extend this study by combining Pelareorep and paclitaxel with the anti-PD-L1 antibody, Avelumab (NCT04215146). In this study, all the therapeutics will be delivered via intravenous infusion. There are many other oncolytic viruses currently undergoing clinical testing in combination with chemotherapy and/or immunotherapy for the treatment of different types of $\mathrm{BrCa}$ (Table 2). Results from these studies are not yet published and it remains to be seen if these combo therapies will result in better treatment of $\mathrm{BrCa}$.

\section{Oncolytic viruses as immunotherapeutics}

Up until a decade ago, the immune system was considered one of the biggest hurdles to the success of oncolytic virotherapy $[67,68]$. This perspective of considering the immune system as a foe of $\mathrm{OV}$ was based on the premise that the main mechanism through which OVs exert anti-tumor effect is through direct lysis of tumor cells by the virtue of virus replication, and hence the anti-viral immune effect is detrimental to the success of OV $[67,68]$. Therefore, in order to maximize the anti-tumor effect of OVs, investigators used a variety of ways to blunt the immune system during $\mathrm{OV}$ therapies. For example, many studies have combined the immunosuppressive drug cyclophosphamide with oncolytic viruses to enhance oncolysis [69-71].

However, recently there has been a shift in the paradigm as an increasing body of evidence show that integral to the success of an oncolytic virus is their ability to elicit antitumor immunity. A seminal study published by Prestwich et al. [72] provided compelling evidence for the immune system as a prerequisite for the success of an oncolytic virus. In this study, the authors demonstrated that oncolytic reovirus could purge lymph nodes and splenic metastases from murine melanoma cell line B16Ova, a line that is unresponsive to virus-mediated cytotoxicity in vitro, in immune-competent mice but not in mice with the compromised immune system. The authors concluded that virusmediated immune activation, but not virus-mediated oncolysis, was critical for the anti-tumor efficacy of the virus. Many other studies have validated this finding using a different type of viruses in a variety of tumor models [7380]. The fact that virus-mediated immune activation is more important than virus-mediated oncolysis was further demonstrated in a recent study by Dai and colleagues [81]. In this study, the authors showed that repeated intratumoral injection of a non-replicating heat-inactivated modified VACV strain Ankara could eradicate different aggressive tumors in mice.

There are several mechanisms through which OVs could activate the immune system against tumor. First, OVmediated oncolysis causes the release of damage-associated molecular patterns (DAMPs) and pathogen-associated molecular patterns (PAMPs) together with a natural repertoire of tumor-specific (TSA) or tumor-associated (TAA) antigens [82, 83] (Fig. 1). Together DAMPs/PAMPs and TAA/TSA provide the key signals to dendritic cells to initiate tumor-specific adaptive immune response [84]. Second, anti-viral immune response within the tumor microenvironment (TME) can convert immunologically "cold" tumors to immunologically "hot" tumors $[85,86]$. We and others have shown that intratumoral injection of oncolytic poxvirus increases pro-inflammatory cytokines such as IFN-y and IL-6 within TME without significantly altering the systemic levels of those cytokines in $\mathrm{BrCa}$ models $[87,88]$. Third, OVs induce immunogenic death in cancer cells $[82,89]$. Mechanisms of cancer cell death elicited by anti-cancer therapeutics are thought to have a decisive role in whether an anti-inflammatory response or anti-tumor response ensues the therapy [90]. Most types of OVs have been shown to induce immunogenic cell death in cancer cells and hence are believed to elicit an anti-tumor immune response (reviewed in ref. [82]). Lastly, since OVs preferentially infect tumor cells, anti-viral immune cells attacking virus-infected cancer cells also clear the cancer cells in the process of clearing OVs [80]. Taken together, 
Fig. 1 Mechanism of action of oncolytic viruses. Oncolytic viruses replicate in tumor cells, induce immunogenic cell death, and spread within the tumor. Viruses released from the injected tumors can then disseminate to distant tumors and infect them. Also, immunogenic cell death induced by oncolytic viruses stimulates anti-tumor immunity that can clear metastatic tumors.

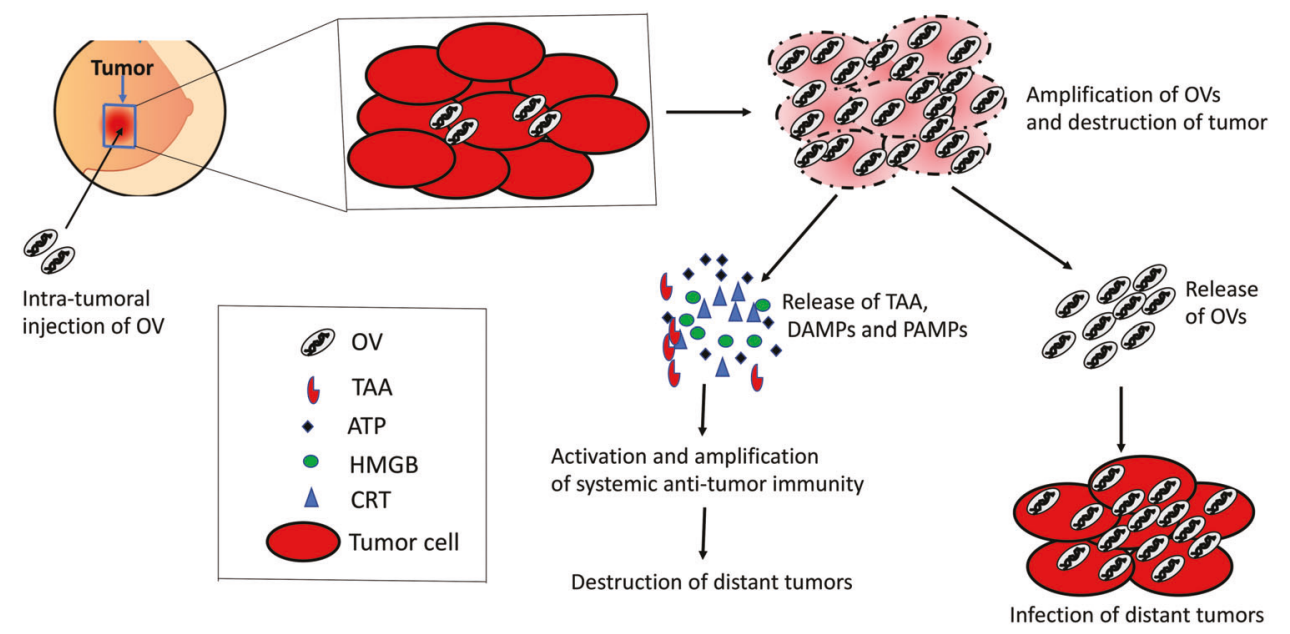

oncolytic viruses are rightfully considered a form of immunotherapy, and the engagement of functional immunity is necessary to harness the optimal anti-tumor efficacy of oncolytic viruses.

The immune-activating feature of OVs can potentiate the use of OVs in neoadjuvant settings for the treatment of BrCa. Bourgeois-Daigneault et al. [61] studied an oncolytic Maraba virus in a neoadjuvant setting for the treatment of murine models of TNBC representative of advanced human TNBC. The authors used three syngeneic orthotopic models (4T1, EMT6, and E0771) all of which form metastases to the lungs, liver, brain, and bones. With the intent of mimicking the course of treatment for women with newly diagnosed TNBC, the authors treated murine tumors intratumorally with oncolytic Maraba virus, surgically resected the tumors 3 days post-treatment, and euthanized the mice 25 days later to determine the number and extent of metastases. Interestingly, in the 4T1 model, 50\% of virustreated mice were found to be metastasis-free, whereas all control-treated mice had metastases in their lungs, and the virus-treated mice that displayed metastasis had fewer and smaller internal and surface lung metastases compared to the control-treated mice. Similar results were demonstrated for EMT6 and EO771 models. Given the fact that metastasis is the main cause of deaths in TNBC patients, the results from this study has profound implications regarding the treatment of TNBC patients. The authors noted that two of the three models used in this study, 4T1 and EMT6, are refractory to Maraba treatment in vivo, which further underscores the importance of the immune-stimulating feature of OVs in the treatment of tumors. Building on this study, Martin et al. [91] further characterized the usage of oncolytic VSV, adenovirus, reovirus and HSV as presurgical neoadjuvant therapy in a murine TNBC model. In this study, subcutaneous 4T1 tumors were treated intratumorally with the virus, and tumors were surgically removed 1 week after first virus injection. Four days after resection, 4T1 cells were re-injected in the mammary fat pad of the mice, and tumor growth as well as survival of mice were followed. In accordance with the study by Bourgeois-Daigneault et al., this study found that most of the studied viruses were able to confer survival benefits in this neoadjuvant setting even though the mice were not treated with anything after re-challenge with the 4T1 cells. Taken together, the ability of OVs to induce immune activation is the most important aspect in the treatment of $\mathrm{BrCa}$.

\section{Combination of virotherapy and immunotherapy}

In general, cancer is a complex disease and a single therapeutic agent will not be enough to completely cure the disease. Therefore, a multi-pronged attack on cancer using a combination of two or more therapeutics is desired. One of the most commonly studied strategies to boost the antitumor efficacy of virotherapy is to combine it with immunotherapy. Immunotherapy is either combined with virotherapy as a separate therapeutic entity or viruses are modified to encode immunotherapeutics, which serves as both virotherapy and immunotherapy. Many replicating and non-replicating viruses have been engineered to encode cytokines and studied for the treatment of $\mathrm{BrCa}$. For example, Ghouse et al. [54] engineered an oncolytic HSV to encode the cytokine IL-12 and studied the anti-tumor efficacy of this virus in murine models of TNBC. The virus was found to increase tumor infiltration by CD45 and $\mathrm{CD}^{+}$ cells and it exerted local and abscopal anti-tumor effects in a syngeneic murine model of TNBC. Another study by Zhu et al. [92] showed that an oncolytic adenovirus armed with the cytokine IL-24 could potently inhibit the growth of breast cancer cells in vitro and in mice. Likewise, Bramante et al. [93] found that an oncolytic adenovirus encoding GMCSF in combination with low dose cyclophosphamide 
exerts anti-tumor efficacy against TNBC in the murine model as well as in human patients.

Because OVs can convert tumors from immunologically "cold" to "hot", it is plausible that the combination of OVs with therapeutics that require "hot" TME, can potentially yield synergistic anti-tumor efficacies. Many studies have shown that while OVs increase tumor infiltration by cytotoxic $\mathrm{T}$ cells and other immune cells, cancer cells also upregulate expression of the checkpoint protein PD-L1 in response to OV therapy [87, 94]. This upregulation in checkpoint protein blocks cytotoxic $\mathrm{T}$ cells from killing the cancer cells. An analogy for this situation would be pressing the gas in a motor car while simultaneously applying the brakes. In order to achieve optimal anti-neoplastic benefits from OV therapy, the brakes also need to be released by using immune checkpoint inhibitors (ICIs). Based on this hypothesis, several preclinical and clinical studies have combined OVs with ICIs for the treatment of different malignancies. For $\mathrm{BrCa}$, the study by Bourgeois-Daigneault et al. [61], which is discussed above, determined if OVs could sensitize TNBC to ICIs. The authors found that oncolytic Maraba was able to sensitize otherwise refractory TNBC to ICIs and prevented disease relapse in the majority of treated mice. Likewise, Mostafa et al. [88] studied the combination of oncolytic reovirus with an antibody against PD-1 in BrCa models. The authors reported that reovirus induced upregulation of PD-L1 levels on tumor cells and increased immune cell infiltration in tumors, and the combination therapy resulted in significantly higher anti-tumor efficacy than either of the monotherapy. In line with these studies, we have recently shown that an oncolytic chimeric poxvirus causes upregulation of PD-L1 expression on $\mathrm{BrCa}$ cells in vitro and in vivo in mice and convert immunologically "cold" TME into "hot". Furthermore, the overall anti-tumor efficacy of the virus was improved by combination with anti-PD-L1 antibody [87]. Tables 1 and 2 list some preclinical and clinical studies, respectively, combining OVs with ICIs for $\mathrm{BrCa}$ therapy.

\section{Challenges in virotherapy for breast cancer}

While virotherapy holds a great promise for better treatment of $\mathrm{BrCa}$, there are some challenges that need to be overcome before the real benefits of this novel therapeutic approach could be realized in patients. Like other solid tumors, challenges associated with virotherapy of $\mathrm{BrCa}$ are mostly related to optimal delivery and spread of the virus as well as therapeutic efficacy. While most clinical studies have found oncolytic viruses to be safe in humans, OVmediated anti-tumor efficacy has been moderate at best. In most cases, the dose-limiting toxicity has not been achieved due to manufacturing constrains [95]. One way to circumvent the manufacturing constrains could be to generate oncolytic viruses with higher tumor-killing potency such that a low dose of viruses can achieve meaningful antitumor efficacy. In this regard, we and others have recently shown that it is possible to create chimeric viruses, using different strains/species of viruses, with enhanced antitumor potency. We generated a chimeric poxvirus (CF33) through recombination among different strains/species of poxvirus that showed superior potential in killing tumor cells compared to the parental viruses in vitro. In vivo, the virus was able to completely control the growth of TNBC xenografts at a dose 100 to 1000 folds lower than that reported for other oncolytic poxviruses [96]. Similarly, Ricordel et al. [97] generated a chimeric poxvirus with improved oncolytic potency through chimerization among different strains of the vaccinia virus.

Optimal delivery of virus to target tumors is difficult to achieve especially for the metastasized disease. Most preclinical studies have used local/regional delivery of virus to individual tumors; however, this is a simplistic approach, which may not be feasible for metastatic lesions that are inaccessible for injection. Systemic delivery of virus is hampered by many factors including virus sequestration in the liver and spleen, virus neutralization by serum factors, and the inability of viruses to cross the endothelial cells lining tumor vasculature [45]. In a phase I clinical trial with an oncolytic vaccinia virus, JX594, it was found that recovery of virus from tumors following systemic injection was possible only when the viremic threshold dose of $10^{9}$ infectious units was exceeded [98]. While this study did not include BrCa patients, it serves as an important example of the enormous amount of virus needed for systemic delivery for the virus to reach tumors. For many types of OVs, manufacturing constraints make it impracticable to use such high doses. Furthermore, the spread of virus within the tumor is hindered by the dense stromal components including the extracellular matrix [99]. Several strategies have been studied to circumvent these hindrances and enhance virus spread in the tumors as discussed in an excellent review article by Jordi and collegues [100]. Although these strategies have shown promising results in preclinical studies, it remains to be seen if they prove to be effective in clinical studies.

\section{Conclusion and future directions}

With the advancements in molecular biology and virology, there has been an ever-increasing interest in using viruses as cancer therapeutics. Preclinical and clinical studies have demonstrated an excellent safety profile of virotherapy in human patients, and strategies to improve anti-tumor efficacy are being extensively studied. Although $\mathrm{BrCa}$ was thought to be less amenable to immunotherapy in past, the recent approval of atezolizumab for the treatment of 
metastatic TNBC has made it clear that $\mathrm{BrCa}$ patients can also benefit from immunotherapy. Furthermore, results from many preclinical studies suggest that the efficacy of immunotherapies in $\mathrm{BrCa}$ can be greatly enhanced by combination with other treatment such as oncolytic virus, which can favorably modulate the tumor-immune landscape. Indeed, many clinical trials are underway testing the combination of immunotherapeutics and oncolytic viruses in BrCa patients. While there is not enough clinical data to conclude the combined effect of oncolytic virus and immunotherapy in the treatment of BrCa patients, based on the preclinical studies and the strong rational of the combination it is likely that the combination may result in synergistic or, at least, additive therapeutic benefits.

Future studies should focus on the improvement of the delivery system for oncolytic viruses. Also, the immuneactivating properties of oncolytic viruses need to be further improved in order to achieve a higher therapeutic benefit. However, the virus-mediated immune activation should be targeted against tumor because general immune activation by the oncolytic virus, especially in combination with checkpoint inhibitors, has the potential of causing severe auto-immune disorders. Recently, investigators are studying if it is possible to achieve tumor-specific immune activation by arming oncolytic viruses with tumor antigen. McGray et al. [101] recently published a study for eliciting tumor antigen-specific immunity using oncolytic Maraba virus armed with tumor antigen. Another avenue to explore would be the combination of an oncolytic virus with chimeric antigen receptor $\mathrm{T}$ (CAR-T) cells in which the target for CAR-T could be delivered by the oncolytic virus. This approach may particularly be more interesting for TNBC, which lack therapeutic targets. Taken together, further studies rationally combining oncolytic viruses with other therapeutics may find the best combination to achieve higher therapeutic efficacy with minimal toxicity for the treatment of $\mathrm{BrCa}$ patients.

Acknowledgements Shyambabu Chaurasiya is supported through the generosity of the Natalie and David Roberts Family. We wish to thank them for their philanthropy.

\section{Compliance with ethical standards}

Conflict of interest The authors declare that they have no conflict of interest.

Publisher's note Springer Nature remains neutral with regard to jurisdictional claims in published maps and institutional affiliations.

\section{References}

1. Bray F, Ferlay J, Soerjomataram I, Siegel RL, Torre LA, Jemal A. Global cancer statistics 2018: GLOBOCAN estimates of incidence and mortality worldwide for 36 cancers in 185 countries. CA Cancer J Clin. 2018;68:394-424.

2. Higgins MJ, Wolff AC. Therapeutic options in the management of metastatic breast cancer. Oncology. 2008;22:614-23.

3. Lu J, Steeg PS, Price JE, Krishnamurthy S, Mani SA, Reuben J, et al. Breast cancer metastasis: challenges and opportunities. Cancer Res. 2009;69:4951-3.

4. Polyak K. Breast cancer: origins and evolution. J Clin Invest. 2007;117:3155-63.

5. Malhotra GK, Zhao X, Band H, Band V. Histological, molecular and functional subtypes of breast cancers. Cancer Biol Ther. 2010;10:955-60.

6. Cancer Genome Atlas Network. Comprehensive molecular portraits of human breast tumours. Nature. 2012;490:61-70.

7. Perou CM, Sorlie T, Eisen MB, van de Rijn M, Jeffrey SS, Rees CA, et al. Molecular portraits of human breast tumours. Nature. 2000;406:747-52.

8. Sorlie T, Wang Y, Xiao C, Johnsen H, Naume B, Samaha RR, et al. Distinct molecular mechanisms underlying clinically relevant subtypes of breast cancer: gene expression analyses across three different platforms. BMC Genomics. 2006;7:127.

9. Sorlie T, Perou CM, Tibshirani R, Aas T, Geisler S, Johnsen H, et al. Gene expression patterns of breast carcinomas distinguish tumor subclasses with clinical implications. Proc Natl Acad Sci USA. 2001;98:10869-74.

10. Burstein HJ, Morrow M. Nodal irradiation after breast-cancer surgery in the era of effective adjuvant therapy. N. Engl J Med. 2015;373:379-81.

11. Fisher B, Jeong JH, Anderson S, Bryant J, Fisher ER, Wolmark N. Twenty-five-year follow-up of a randomized trial comparing radical mastectomy, total mastectomy, and total mastectomy followed by irradiation. N Engl J Med. 2002;347:567-75.

12. Cotlar AM, Dubose JJ, Rose DM. History of surgery for breast cancer: radical to the sublime. Curr Surg. 2003;60:329-37.

13. Anampa J, Makower D, Sparano JA. Progress in adjuvant chemotherapy for breast cancer: an overview. BMC Med. 2015;13:195.

14. White J, Achuthan R, Turton P, Lansdown M. Breast conservation surgery: state of the art. Int J Breast Cancer. 2011;2011: 107981.

15. Jin $\mathrm{X}, \mathrm{Mu} \mathrm{P}$. Targeting breast cancer metastasis. Breast Cancer (Auckl). 2015;9:23-34.

16. Gupta GP, Massague J. Cancer metastasis: building a framework. Cell 2006;127:679-95.

17. Ciccone E, Viale O, Bottino C, Pende D, Migone N, Casorati G, et al. Antigen recognition by human T-cell receptor positive-gamma lymphocytes - specific lysis of allogeneic cells after activation in mixed lymphocyte culture. J Exp Med. 1988;167:1517-22.

18. Hudis CA, Dang CT. Adjuvant therapy for breast cancer: practical lessons from the early breast cancer trialists' collaborative group. Breast Dis. 2004;21:3-13.

19. Clarke M. Meta-analyses of adjuvant therapies for women with early breast cancer: the Early Breast Cancer Trialists' Collaborative Group overview. Ann Oncol. 2006;17:x59-62.

20. Vallis KA, Tannock IF. Postoperative radiotherapy for breast cancer: growing evidence for an impact on survival. J Natl Cancer Inst. 2004;96:88-9.

21. Nabholtz JM, Gligorov J. The role of taxanes in the treatment of breast cancer. Expert Opin Pharmacother. 2005;6:1073-94.

22. Jones SE. Metastatic breast cancer: the treatment challenge. Clin Breast Cancer. 2008;8:224-33.

23. Early Breast Cancer Trialists' Collaborative Group. Effects of chemotherapy and hormonal therapy for early breast cancer on recurrence and 15-year survival: an overview of the randomised trials. Lancet. 2005;365:1687-717. 
24. Zhao M, Ramaswamy B. Mechanisms and therapeutic advances in the management of endocrine-resistant breast cancer. World $\mathbf{J}$ Clin Oncol. 2014;5:248-62.

25. Slamon D, Eiermann W, Robert N, Pienkowski T, Martin M, Press M, et al. Adjuvant trastuzumab in HER2-positive breast cancer. N. Engl J Med. 2011;365:1273-83.

26. Baselga J, Perez EA, Pienkowski T, Bell R. Adjuvant trastuzumab: a milestone in the treatment of HER-2-positive early breast cancer. Oncologist 2006;11:4-12.

27. Jelovac D, Wolff AC. The adjuvant treatment of HER2-positive breast cancer. Curr Treat Options Oncol. 2012;13:230-9.

28. Anders C, Carey LA. Understanding and treating triple-negative breast cancer. Oncology. 2008;22:1233-9.

29. Carey LA, Dees EC, Sawyer L, Gatti L, Moore DT, Collichio F, et al. The triple negative paradox: primary tumor chemosensitivity of breast cancer subtypes. Clin Cancer Res. 2007;13:2329-34.

30. Hanahan D, Weinberg RA. Hallmarks of cancer: the next generation. Cell 2011;144:646-74.

31. Cross D, Burmester JK. Gene therapy for cancer treatment: past, present and future. Clin Med Res. 2006;4:218-27.

32. Zarogoulidis P, Darwiche K, Sakkas A, Yarmus L, Huang H, Li Q, et al. Suicide Gene Therapy for Cancer - Current Strategies. J Genet Syndr Gene Ther. 2013;4:16849.

33. Majumdar AS, Zolotorev A, Samuel S, Tran K, Vertin B, HallMeier M, et al. Efficacy of herpes simplex virus thymidine kinase in combination with cytokine gene therapy in an experimental metastatic breast cancer model. Cancer Gene Ther. 2000;7:1086-99.

34. Brockstedt DG, Diagana M, Zhang Y, Tran K, Belmar N, Meier $\mathrm{M}$, et al. Development of anti-tumor immunity against a nonimmunogenic mammary carcinoma through in vivo somatic GMCSF, IL-2, and HSVtk combination gene therapy. Mol Ther. 2002;6:627-36.

35. Pandha HS, Martin LA, Rigg A, Hurst HC, Stamp GW, Sikora $\mathrm{K}$, et al. Genetic prodrug activation therapy for breast cancer: A phase I clinical trial of erbB-2-directed suicide gene expression. J Clin Oncol. 1999;17:2180-9.

36. Braybrooke JP, Slade A, Deplanque G, Harrop R, Madhusudan $\mathrm{S}$, Forster MD, et al. Phase I study of MetXia-P450 gene therapy and oral cyclophosphamide for patients with advanced breast cancer or melanoma. Clin Cancer Res. 2005;11:1512-20.

37. Zheng L, Weilun Z, Minghong J, Yaxi Z, Shilian L, Yanxin L, et al. Adeno-associated virus-mediated doxycycline-regulatable TRAIL expression suppresses growth of human breast carcinoma in nude mice. BMC Cancer. 2012;12:153.

38. Li YM, Wen Y, Zhou BP, Kuo HP, Ding Q, Hung MC. Enhancement of Bik antitumor effect by Bik mutants. Cancer Res. 2003;63:7630-3.

39. Bargou RC, Wagener C, Bommert K, Mapara MY, Daniel PT, Arnold $\mathrm{W}$, et al. Overexpression of the death-promoting gene bax-alpha which is downregulated in breast cancer restores sensitivity to different apoptotic stimuli and reduces tumor growth in SCID mice. J Clin Invest. 1996;97:2651-9.

40. Rakkar AN, Katayose Y, Kim M, Craig C, Ohri E, Li Z, et al. A novel adenoviral vector expressing human Fas/CD95/APO-1 enhances p53-mediated apoptosis. Cell Death Differ. 1999;6:326-33.

41. Ogawa T, Kusumoto M, Mizumoto K, Sato N, Tanaka M. Adenoviral GM-CSF gene transduction into breast cancer cells induced long-lasting antitumor immunity in mice. Breast Cancer. 1999;6:301-4.

42. Chaurasiya S, Hew P, Crosley P, Sharon D, Potts K, Agopsowicz $\mathrm{K}$, et al. Breast cancer gene therapy using an adenovirus encoding human IL-2 under control of mammaglobin promoter/ enhancer sequences. Cancer Gene Ther. 2016;23:178-87.

43. Divino CM, Chen SH, Yang W, Thung S, Brower ST, Woo SL. Anti-tumor immunity induced by interleukin-12 gene therapy in a metastatic model of breast cancer is mediated by natural killer cells. Breast Cancer Res Treat. 2000;60:129-34.

44. Lapteva N, Aldrich M, Weksberg D, Rollins L, Goltsova T, Chen SY, et al. Targeting the intratumoral dendritic cells by the oncolytic adenoviral vaccine expressing RANTES elicits potent antitumor immunity. J Immunother. 2009;32:145-56.

45. Russell SJ, Peng KW, Bell JC. Oncolytic virotherapy. Nat Biotechnol. 2012;30:658-70.

46. Greig SL. Talimogene laherparepvec: first global approval. Drugs 2016;76:147-54.

47. Yamamoto M, Curiel DT. Current issues and future directions of oncolytic adenoviruses. Mol Ther. 2010;18:243-50.

48. Bauerschmitz GJ, Ranki T, Kangasniemi L, Ribacka C, Eriksson M, Porten M, et al. Tissue-specific promoters active in CD44+CD24-/low breast cancer cells. Cancer Res. 2008;68:5533-9.

49. Liikanen I, Tahtinen S, Guse K, Gutmann T, Savola P, Oksanen M, et al. Oncolytic adenovirus expressing monoclonal antibody trastuzumab for treatment of HER2-positive cancer. Mol Cancer Ther. 2016;15:2259-69.

50. Xu W, Yang Y, Hu Z, Head M, Mangold KA, Sullivan M, et al. LyP-1-modified oncolytic adenoviruses targeting transforming growth factor beta inhibit tumor growth and metastases and augment immune checkpoint inhibitor therapy in breast cancer mouse models. Hum Gene Ther. 2020;31:15-6.

51. Akhtar J, Shukla D. Viral entry mechanisms: cellular and viral mediators of herpes simplex virus entry. FEBS J. 2009;276:7228-36.

52. Menotti L, Cerretani A, Hengel H, Campadelli-Fiume G. Construction of a fully retargeted herpes simplex virus 1 recombinant capable of entering cells solely via human epidermal growth factor receptor 2. J Virol. 2008;82:10153-61.

53. Leoni V, Gatta V, Palladini A, Nicoletti G, Ranieri D, Dall'Ora $\mathrm{M}$, et al. Systemic delivery of HER2-retargeted oncolytic-HSV by mesenchymal stromal cells protects from lung and brain metastases. Oncotarget 2015;6:34774-87.

54. Ghouse SM, Nguyen HM, Bommareddy PK, Guz-Montgomery K, Saha D. Oncolytic Herpes Simplex virus encoding IL12 controls triple-negative breast cancer growth and metastasis. Front Oncol. 2020;10:384.

55. Gholami S, Marano A, Chen NG, Aguilar RJ, Frentzen A, Chen $\mathrm{CH}$, et al. A novel vaccinia virus with dual oncolytic and antiangiogenic therapeutic effects against triple-negative breast cancer. Breast Cancer Res Treat. 2014;148:489-99.

56. Ferguson MS, Chard Dunmall LS, Gangeswaran R, Marelli G, Tysome JR, Burns E, et al. Transient inhibition of PI3Kdelta enhances the therapeutic effect of intravenous delivery of oncolytic vaccinia virus. Mol Ther. 2020;28:1263-75.

57. Chon HJ, Lee WS, Yang H, Kong SJ, Lee NK, Moon ES, et al. Tumor microenvironment remodeling by intratumoral oncolytic vaccinia virus enhances the efficacy of immune-checkpoint blockade. Clin Cancer Res. 2019;25:1612-23.

58. Umer BA, Noyce RS, Franczak BC, Shenouda MM, Kelly RG, Favis NA, et al. Deciphering the immunomodulatory capacity of oncolytic vaccinia virus to enhance the immune response to breast cancer. Cancer Immunol Res. 2020;8:618-31.

59. Niavarani SR, Lawson C, Boudaud M, Simard C, Tai LH. Oncolytic vesicular stomatitis virus-based cellular vaccine improves triple-negative breast cancer outcome by enhancing natural killer and CD8(+) T-cell functionality. J Immunother Cancer. 2020;8:e000465.

60. Arulanandam R, Taha Z, Garcia V, Selman M, Chen A, Varette $\mathrm{O}$, et al. The strategic combination of trastuzumab emtansine with oncolytic rhabdoviruses leads to therapeutic synergy. Commun Biol. 2020;3:254.

61. Bourgeois-Daigneault MC, Roy DG, Aitken AS, El Sayes N, Martin NT, Varette O, et al. Neoadjuvant oncolytic virotherapy 
before surgery sensitizes triple-negative breast cancer to immune checkpoint therapy. Sci Transl Med. 2018;10:eaao1641.

62. Bourgeois-Daigneault MC, St-Germain LE, Roy DG, Pelin A, Aitken AS, Arulanandam R, et al. Combination of Paclitaxel and MG1 oncolytic virus as a successful strategy for breast cancer treatment. Breast Cancer Res. 2016;18:83.

63. Mohamed Amin Z, Che Ani MA, Tan SW, Yeap SK, Alitheen NB, Syed Najmuddin SUF, et al. Evaluation of a recombinant newcastle disease virus expressing human IL12 against human breast cancer. Sci Rep. 2019;9:13999.

64. Rodriguez Stewart RM, Berry JTL, Berger AK, Yoon SB, Hirsch AL, Guberman JA, et al. Enhanced Killing of Triple-Negative Breast Cancer Cells by Reassortant Reovirus and Topoisomerase Inhibitors. J Virol. 2019;93:e1411-19.

65. Sahin TT, Kasuya H, Nomura N, Shikano T, Yamamura K, Gewen T, et al. Impact of novel oncolytic virus HF10 on cellular components of the tumor microenviroment in patients with recurrent breast cancer. Cancer Gene Ther. 2012;19:229-37.

66. Bernstein V, Ellard SL, Dent SF, Tu D, Mates M, Dhesy-Thind SK, et al. A randomized phase II study of weekly paclitaxel with or without pelareorep in patients with metastatic breast cancer: final analysis of Canadian Cancer Trials Group IND.213. Breast Cancer Res Treat. 2018;167:485-93.

67. Parato KA, Senger D, Forsyth PA, Bell JC. Recent progress in the battle between oncolytic viruses and tumours. Nat Rev Cancer. 2005;5:965-76.

68. Filley AC, Dey M. Immune system, friend or foe of oncolytic virotherapy? Front Oncol. 2017;7:106.

69. Atezolizumab for the treatment of breast cancer. PMID: 32067545 .

70. Wakimoto H, Fulci G, Tyminski E, Chiocca EA. Altered expression of antiviral cytokine mRNAs associated with cyclophosphamide's enhancement of viral oncolysis. Gene Ther. 2004;11:214-23.

71. Fulci G, Breymann L, Gianni D, Kurozomi K, Rhee SS, Yu J, et al. Cyclophosphamide enhances glioma virotherapy by inhibiting innate immune responses. Proc Natl Acad Sci USA. 2006;103:12873-8.

72. Prestwich RJ, Ilett EJ, Errington F, Diaz RM, Steele LP, Kottke $\mathrm{T}$, et al. Immune-mediated antitumor activity of reovirus is required for therapy and is independent of direct viral oncolysis and replication. Clin Cancer Res. 2009;15:4374-81.

73. Apostolidis L, Schirrmacher V, Fournier P. Host mediated antitumor effect of oncolytic Newcastle disease virus after locoregional application. Int J Oncol. 2007;31:1009-19.

74. Kleijn A, Kloezeman J, Treffers-Westerlaken E, Fulci G, Leenstra S, Dirven C, et al. The therapeutic efficacy of the oncolytic virus Delta24-RGD in a murine glioma model depends primarily on antitumor immunity. Oncoimmunology 2014;3:e955697.

75. Leddon JL, Chen CY, Currier MA, Wang PY, Jung FA, Denton NL, et al. Oncolytic HSV virotherapy in murine sarcomas differentially triggers an antitumor T-cell response in the absence of virus permissivity. Mol Ther Oncolytics. 2015;1:14010.

76. Bridle BW, Clouthier D, Zhang L, Pol J, Chen L, Lichty BD, et al. Oncolytic vesicular stomatitis virus quantitatively and qualitatively improves primary $\mathrm{CD} 8(+) \mathrm{T}$-cell responses to anticancer vaccines. Oncoimmunology. 2013;2:e26013.

77. Li H, Dutuor A, Fu X, Zhang X. Induction of strong antitumor immunity by an HSV-2-based oncolytic virus in a murine mammary tumor model. J Gene Med. 2007;9:161-9.

78. Miller CG, Fraser NW. Requirement of an integrated immune response for successful neuroattenuated HSV-1 therapy in an intracranial metastatic melanoma model. Mol Ther. 2003;7:741-7.
79. Qiao J, Kottke T, Willmon C, Galivo F, Wongthida P, Diaz RM, et al. Purging metastases in lymphoid organs using a combination of antigen-nonspecific adoptive $\mathrm{T}$ cell therapy, oncolytic virotherapy and immunotherapy. Nat Med. 2008;14:37-44.

80. Gujar S, Pol JG, Kim Y, Lee PW, Kroemer G. Antitumor benefits of antiviral immunity: an underappreciated aspect of oncolytic virotherapies. Trends Immunol. 2018;39:209-21.

81. Dai P, Wang W, Yang N, Serna-Tamayo C, Ricca JM, Zamarin $\mathrm{D}$, et al. Intratumoral delivery of inactivated modified vaccinia virus Ankara (iMVA) induces systemic antitumor immunity via STING and Batf3-dependent dendritic cells. Sci Immunol. 2017;2:eaal1713.

82. Guo ZS, Liu Z, Bartlett DL. Oncolytic immunotherapy: dying the right way is a key to eliciting potent antitumor immunity. Front Oncol. 2014;4:74.

83. Chaurasiya S, Chen NG, Fong Y. Oncolytic viruses and immunity. Curr Opin Immunol. 2018;51:83-90.

84. Tang D, Kang R, Coyne CB, Zeh HJ, Lotze MT. PAMPs and DAMPs: signal 0s that spur autophagy and immunity. Immunol Rev. 2012;249:158-75.

85. Ribas A, Dummer R, Puzanov I, VanderWalde A, Andtbacka RHI, Michielin O, et al. Oncolytic virotherapy promotes intratumoral $\mathrm{T}$ cell infiltration and improves anti-PD-1 immunotherapy. Cell 2017;170:1109-19.e10.

86. Gujar S, Pol JG, Kroemer G. Heating it up: oncolytic viruses make tumors 'hot' and suitable for checkpoint blockade immunotherapies. Oncoimmunology. 2018;7:e1442169.

87. Chaurasiya S, Yang A, Kang S, Lu J, Kim SI, Park AK, et al. Oncolytic poxvirus CF33-hNIS-DeltaF14.5 favorably modulates tumor immune microenvironment and works synergistically with anti-PD-L1 antibody in a triple-negative breast cancer model. Oncoimmunology. 2020;9:1729300.

88. Mostafa AA, Meyers DE, Thirukkumaran CM, Liu PJ, Gratton $\mathrm{K}$, Spurrell J, et al. Oncolytic reovirus and immune checkpoint inhibition as a novel immunotherapeutic strategy for breast cancer. Cancers. 2018;10:205.

89. Workenhe ST, Mossman KL. Oncolytic virotherapy and immunogenic cancer cell death: sharpening the sword for improved cancer treatment strategies. Mol Ther. 2014;22:251-6.

90. Kroemer G, Galluzzi L, Kepp O, Zitvogel L. Immunogenic cell death in cancer therapy. Annu Rev Immunol. 2013;31:51-72.

91. Martin NT, Roy DG, Workenhe ST, van den Wollenberg DJM, Hoeben RC, Mossman KL, et al. Pre-surgical neoadjuvant oncolytic virotherapy confers protection against rechallenge in a murine model of breast cancer. Sci Rep. 2019;9:1865.

92. Zhu W, Wei L, Zhang H, Chen J, Qin X. Oncolytic adenovirus armed with IL-24 inhibits the growth of breast cancer in vitro and in vivo. J Exp Clin Cancer Res. 2012;31:51.

93. Bramante S, Koski A, Liikanen I, Vassilev L, Oksanen M, Siurala $\mathrm{M}$, et al. Oncolytic virotherapy for treatment of breast cancer, including triple-negative breast cancer. Oncoimmunology. 2016;5:e1078057.

94. Liu Z, Ravindranathan R, Kalinski P, Guo ZS, Bartlett DL. Rational combination of oncolytic vaccinia virus and PD-L1 blockade works synergistically to enhance therapeutic efficacy. Nat Commun. 2017;8:14754.

95. Chaurasiya S, Fong Y, Warner SG. Optimizing oncolytic viral design to enhance antitumor efficacy: progress and challenges. Cancers. 2020;12:1699.

96. Choi AH, O'Leary MP, Lu J, Kim SI, Fong Y, Chen NG. Endogenous Akt activity promotes virus entry and predicts efficacy of novel chimeric orthopoxvirus in triple-negative breast cancer. Mol Ther Oncolytics. 2018;9:22-9. 
97. Ricordel M, Foloppe J, Antoine D, Findeli A, Kempf J, Cordier P, et al. Vaccinia virus shuffling: deVV5, a novel chimeric poxvirus with improved oncolytic potency. Cancers. 2018;10:231.

98. Breitbach CJ, Burke J, Jonker D, Stephenson J, Haas AR, Chow LQ, et al. Intravenous delivery of a multi-mechanistic cancer-targeted oncolytic poxvirus in humans. Nature 2011;477:99-102.

99. Wojton J, Kaur B. Impact of tumor microenvironment on oncolytic viral therapy. Cytokine Growth Factor Rev. 2010;21:127-34.
100. Martinez-Quintanilla J, Seah I, Chua M, Shah K. Oncolytic viruses: overcoming translational challenges. J Clin Invest. 2019;130:1407-18.

101. McGray AJR, Huang RY, Battaglia S, Eppolito C, Miliotto A, Stephenson KB, et al. Oncolytic Maraba virus armed with tumor antigen boosts vaccine priming and reveals diverse therapeutic response patterns when combined with checkpoint blockade in ovarian cancer. J Immunother Cancer. 2019;7:189. 